\title{
A Selective Metal-Free Deoxygenation of Unsymmetrical 1,2-Dicarbonyl Compounds by Chlorotrimethylsilane and Sodium Iodide
}

\author{
Ling-Zhi Yuan, ${ }^{\ddagger}$ Dolor Renko, ${ }^{\ddagger}$ Ilhem Khelifi, Olivier Provot*, Jean-Daniel Brion, Abdallah Hamze, \\ and Mouad Alami*
}

BioCIS, Univ. Paris-Sud, CNRS, équipe labellisée Ligue Contre le Cancer, Université Paris-Saclay, 92290, Châtenay-Malabry, France

Supporting Information Placeholder

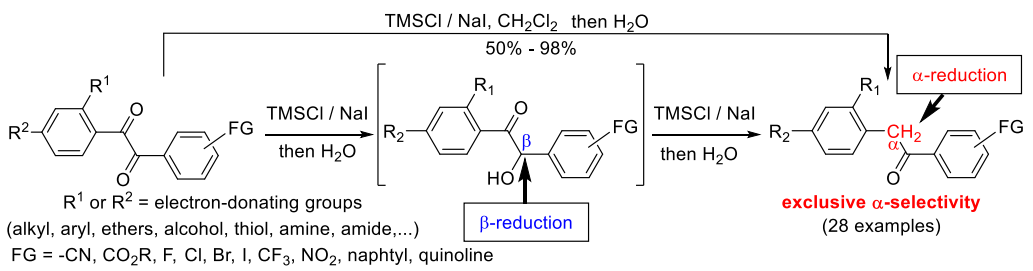

\begin{abstract}
For the first time, the combination of chlorotrimethylsilane (TMSCl) with $\mathrm{NaI}$ is used as a selective reducting system towards 1,2-diketones. This combination is successfully evaluated with several unsymmetrically benzil derivatives, which are reduced in good yields and with a total $\alpha$-regioselectivity at room temperature. Identification of benzoin intermediates is achieved and a mechanistic radical process is proposed.
\end{abstract}

It is well-established that trimethylsilyl iodide (TMSI), in which silicon atom acts as a hard acid and iodide as a soft base, is a useful and powerful reagent in organic synthesis. ${ }^{1}$ TMSI reacts with oxygen-containing compounds forming a strong siliconoxygen bond. The iodide then acts as a strong nucleophile in a subsequent displacement step, thus resulting in cleavage of carbon-oxygen bonds. Typical applications of this reagent are the cleavage of ethers or carboxylic esters, conversion of ketals into carbonyl compounds, and alcohols into iodide derivatives. Most of these transformations occurred through an ionic mechanism. Although ketones readily react with TMSI to provide enol silyl ethers, ${ }^{2}$ to our knowledge, except deoxygenation of sulfoxides into sulfides, ${ }^{3}$ nothing is known about the ability of TMSI to act as a reducing agent with carbonyl compounds.

Carbonyl reduction is among the most important and prevalent reactions in organic synthesis, and both industry as well as academia place special emphasis on this transformation due to its versatility for the generation of a wide range of products. ${ }^{4}$ In this context, the deoxygenation of ketones to methylene derivatives is also an essential process in organic chemistry. ${ }^{5}$ Although relatively uncommon in process chemistry, the reductive mono deoxygenation of 1,2-dicarbonyl compounds into monoketone derivatives has also been reported, but preparative methods that may drive these reactions selectively have been seldom optimized in the past. To accomplish this transformation, the use of catalytic hydrogenation in the presence of $\mathrm{Ni}(0)$ complexes $^{6}$ and metal-based reducing agents $\left(\mathrm{TiCl}_{4} / \mathrm{Zn}, \mathrm{SmI}_{2}\right)^{7}$ comprise most examples, but metal-free procedures $(\mathrm{HI} / \mathrm{AcOH}$ and $\left.\mathrm{H}_{2} \mathrm{~S} / \mathrm{py}\right)^{8}$ have also been reported. From a large-scale pre- parative point of view, these protocols suffer from many drawbacks including the use of flammable gas $\left(\mathrm{H}_{2}\right.$ or $\left.\mathrm{H}_{2} \mathrm{~S}\right)$, the frequent need for specialized equipment, the requirement of harsh reaction conditions, and the low functional group tolerance (chemo- and regioselectivity issues) which limit their application in poly-functionalized substrates. Therefore, the availability of a mild, simple, safe, chemoselective and easy-to-handle reducing agent is of special interest because it allows the formation of complex organic target molecules without the need for wasteful protection/deprotection steps. Herein, we report a highly efficient regioselective reduction of 1,2-diarylketone derivatives using the couple chlorotrimethylsilane $(\mathrm{TMSCl}) / \mathrm{NaI}$ in methylene chloride.

Scheme 1. Regioselective reduction of 1 a by $\mathrm{TMSCl} / \mathrm{NaI}$ in $\mathrm{CH}_{2} \mathrm{Cl}_{2}$ at rt.

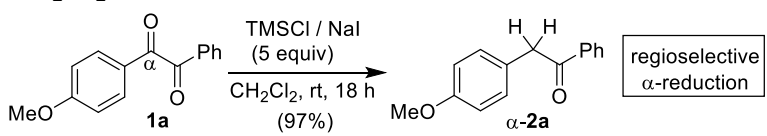

Initially, we had to investigate the ether cleavage of benzil derivative 1a in $\mathrm{CH}_{2} \mathrm{Cl}_{2}$ using an excess of $\mathrm{TMSCl} / \mathrm{NaI}$ combination at room temperature (Scheme 1). Contrary to our expectation, the reaction did not afford the desired phenol derivative. We serendipitously discovered that, under these mild conditions, if the 4-MeO group remained unchanged, one of the two carbonyl functions $(\mathrm{C} \alpha=\mathrm{O})$ of benzil 1a was totally reduced to produce the $\alpha$-deoxybenzoin (DOB) $\alpha-2 \mathbf{a}^{9}$ as a single product $(97 \%)^{10}$ with a total $\alpha$-regioselectivity. A similar yield of $\alpha-2 \mathbf{a}$ $(95 \%)$ was obtained when achieving this reaction under anhydrous conditions (oven-dried $\mathrm{NaI}$ and distillation of TMSCl and 
$\mathrm{CH}_{2} \mathrm{Cl}_{2}$ over $\mathrm{CaH}_{2}$ prior to use). This result suggests that traces of $\mathrm{HCl}$ in technical TMSCl or traces of moisture in the solvent have no influence on the outcome of the reducing properties of the TMSCl$/ \mathrm{NaI}$ combination. To understand this unexpected selective reduction, we initially investigated the reaction of $\mathbf{1 a}$ in $\mathrm{CH}_{2} \mathrm{Cl}_{2}$ with different combinations of silicon species and salts. First, we observed that the reduction process of $\mathbf{1 a}$ did not occur with $\mathrm{TMSCl}$ alone, 1a was recovered unchanged even when achieving the reaction at $40{ }^{\circ} \mathrm{C}$. Replacing TMSCl/NaI combination by TMSI allows the reaction to occur providing $\alpha-2 \mathbf{a}$, but with a lower yield of $65 \%$. Next, we focused our attention on the sources of silicon species in the presence of $\mathrm{NaI}$ in this process. With TMSOTf, no reaction occurred whereas, $\mathrm{SiCl}_{4}$ led to $\alpha$-2a in a modest $65 \%$ yield, along with unidentified byproducts. However, we were pleased to observe that the regioselective reduction of 1 a was still efficient using $\mathrm{Ph}_{3} \mathrm{SiCl}$ $(97 \%)$ or $(\mathrm{EtO})_{3} \mathrm{SiCl}(87 \%)$ in place of $\mathrm{TMSCl}$, but required 10 equiv of the $(\mathrm{EtO})_{3} \mathrm{SiCl} / \mathrm{NaI}$ combination. The nature of the additive salt was also studied and no reduction of 1 a occurred when replacing $\mathrm{NaI}$ by $\mathrm{NaBr}$, clearly indicating that the iodide counter ion is important in this reaction. Because TMSI fumes in air and should be prepared freshly, distillated and immediately used on the contrary of TMSCl, we have chosen to take advantage of this easy to handle and inexpensive TMSCl/NaI combination to reduce benzil derivatives $\mathbf{1}$. Screening with respect to the solvents was next carried out. No reduction reaction occurred in protic polar solvents (e.g., $\mathrm{MeOH}, \mathrm{EtOH}$ or $i \mathrm{PrOH})$ at $\mathrm{rt}$, and 1a was recovered unchanged. The use of aprotic polar solvent (e.g., $\mathrm{CH}_{3} \mathrm{CN}$ ), resulted in a sluggish reaction and $\mathbf{2 a}$ was isolated with a modest $50 \%$ yield after $18 \mathrm{~h}$ of reaction. Among the solvents studied, $\mathrm{CHCl}_{3}, \mathrm{CCl}_{4}$ or toluene have proven to be effective as $\mathrm{CH}_{2} \mathrm{Cl}_{2}$, providing $\alpha$-2a with nearly quantitative yields. Several control experiments were achieved, as suggested by the referees, to prove that the TMSCl/NaI combination is the reducing system rather than small amounts of $\mathrm{HI}^{\mathrm{8a}-\mathrm{c}}$ formed between $\mathrm{TMSCl} / \mathrm{NaI}$ and traces of water present in the solvent. Thus, when performing the reduction of $\mathbf{1 a}$ in $\mathrm{CH}_{2} \mathrm{Cl}_{2}$ at $\mathrm{rt}$ for $24 \mathrm{~h}$ with $0.5,2$ or 10 equiv of aqueous $\mathrm{HI}$ (wt $57 \%$ ), no reduction occurred. Increasing the amount of HI to 15 equiv, resulted in a mixture in which the expected $\alpha-2 \mathbf{a}$ was formed in less than $15 \% .{ }^{11}$ All of these results strongly confirm that the novel reducing agent $\mathrm{TMSCl} / \mathrm{NaI}$ is responsible for this transformation, discarding definitively the HI hypothesis.

After optimizing the experimental conditions with $\mathbf{1 a}$ and TMSCl/NaI combination (5 equiv) in $\mathrm{CH}_{2} \mathrm{Cl}_{2}$, the scope of this reduction reaction was explored with several other benzils $\mathbf{1 b}$ $\mathbf{y}$. As shown in Table 1, a variety of functional groups was well tolerated to give rise to the desired $\alpha$-DOBs 2 with good to excellent yields. Examination of results showed that a total $\alpha$-regioselectivity was observed for several benzils having an electron donating group (EDG) on ortho- and para-position. This regioselective reduction was also effective with a para freephenol derivative providing DOB $\alpha-2 \mathbf{h}$ with a high $89 \%$ yield. One note that $\mathbf{\alpha}-\mathbf{2 h}$ was also obtained from other benzil derivatives bearing on the 4-position a -OMOM $(\alpha-2 \mathbf{h}, 50 \%)$, -OTBS $(\alpha-2 \mathbf{h}, 84 \%)$, -OBn $(\alpha-2 \mathbf{h}, 67 \%)$, and -OAc ( $\alpha-2 \mathbf{h}, 80 \%)$ function. For $p$-ethoxycarbonylbenzil substrate, the reactivity of the diketone significantly decreased, leading to a complex mix-ture with no trace of DOB 2l. However, push-pull benzil derivatives were successfully and regioselectively reduced into the desired $\alpha$-DOBs $\mathbf{2 m - t}$ with excellent yields. At least, an example of re- duction of a push-push $\alpha$-diketone is described, and as expected, DOB $\mathbf{2 y}$ was obtained as a regioisomeric mixture of monoketones $(\alpha-2 y / \beta-2 y=84 / 16)$, indicating that the reduction takes place preferentially on the carbon atom proximal to the more electron-rich ring. To propose a plausible mechanism of this regioselective reduction process of $\alpha$-diketones, we next isolated the benzoin intermediates by quenching the reaction with $\mathrm{H}_{2} \mathrm{O}$ after reduced reaction times and/or temperature (Scheme 2). The reduction of benzils $\mathbf{1 a}$ and $\mathbf{1 b}$ was achieved at $-40{ }^{\circ} \mathrm{C}$ for $15 \mathrm{~min}$ whereas the reduction of quinoline derivative $1 \mathrm{x}$ required $2.5 \mathrm{~h}$ of reaction at $\mathrm{rt}$.

Table 1: Functional groups tolerance ${ }^{\mathrm{a}}$

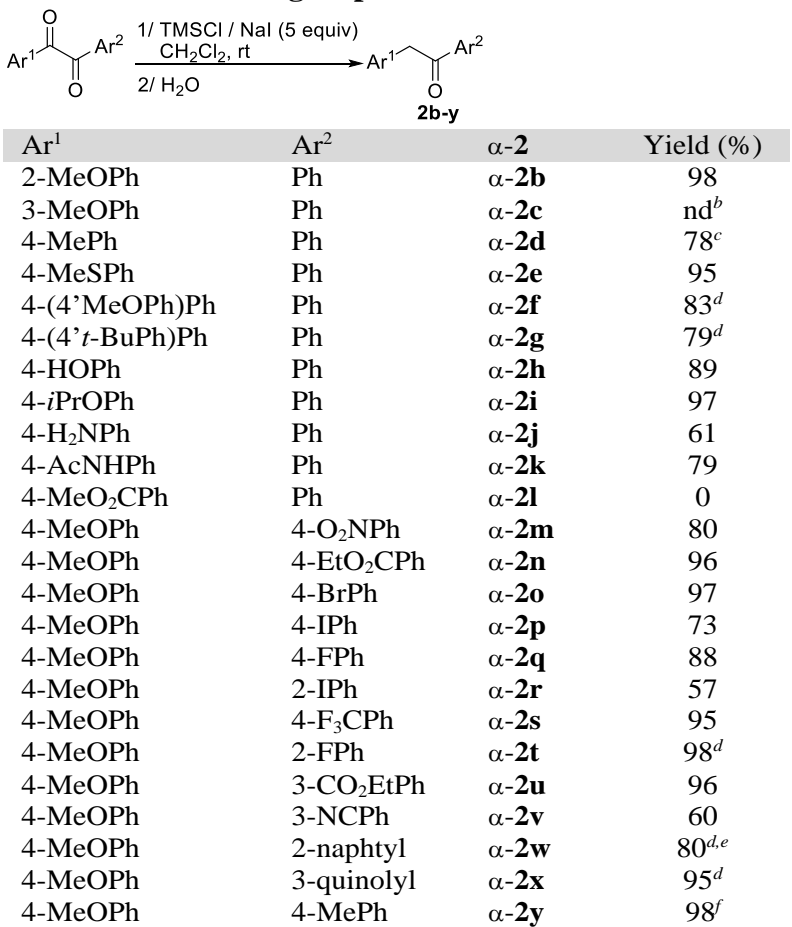

${ }^{a}$ Typical reaction conditions: a mixture of benzil $1(1 \mathrm{mmol})$ and $\mathrm{NaI}$ ( $5 \mathrm{mmol}$ ) was stirred in $\mathrm{CH}_{2} \mathrm{Cl}_{2}$ for $5 \mathrm{~min}$. Then, $5 \mathrm{mmol}$ of TMSCl were added to the solution which was stirred until completion (judged by TLC). ${ }^{b} \mathrm{Nd}$ not determined. ${ }^{c}$ Obtained as an inseparable mixture of $\alpha-2 d / \beta-2 d=88: 12{ }^{d}$ Experiment was achieved in refluxing $\mathrm{CHCl}_{3}$. ${ }^{e}$ Obtained as a separable mixture of $\alpha-2 w / \beta-2 w=90: 10 .{ }^{f}$ Obtained as an inseparable mixture of $\alpha-2 \mathbf{y} / \beta-2 \mathbf{y}=84: 16$.

Scheme 2. Synthesis of DOBs 2 via their benzoin intermediates 3.

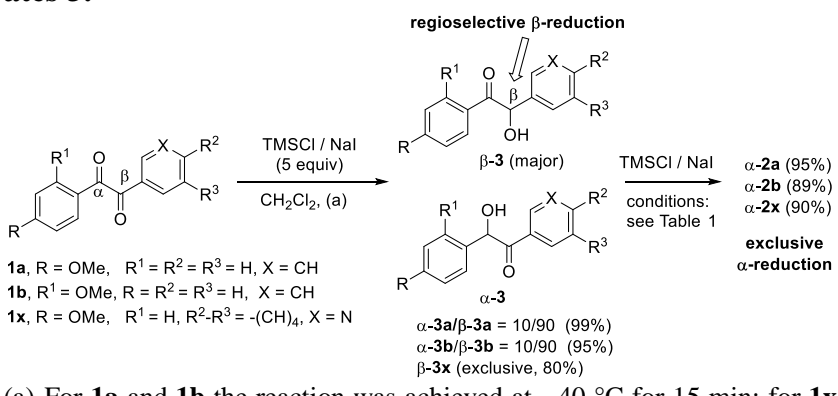

(a) For $1 \mathbf{a}$ and $\mathbf{1 b}$ the reaction was achieved at $-40{ }^{\circ} \mathrm{C}$ for $15 \mathrm{~min}$; for $\mathbf{1 x}$ the reaction was carried out at $\mathrm{rt}$ for $2.5 \mathrm{~h}$.

In all cases and to our surprise, the reduction of these benzils proceeded overwhelmingly on the $\mathrm{C} \beta$ of diketones studied, to furnish mainly $\beta$-benzoins $\mathbf{3 a}$ and $\mathbf{3 b}$ and exclusively $\beta-\mathbf{3} \mathbf{x}$ with good to excellent yields. ${ }^{12}$ 
One can note that a less marked $\beta$-regioselectivity has been previously reported with very few examples of unsymmetrical benzils using other reducing agents such as alkylphosphines, ${ }^{13}$ baker's yeast, ${ }^{14}$ and NADH models. ${ }^{15}$ Next, the reduction of benzoins 3a,b and $\beta-\mathbf{3 x}$ was attempted under the conditions previously reported in Table 1 . To our delight, a "reverse" C $\alpha$-re- duction occurred invariably to furnish the previously obtained $\alpha$-DOBs $\mathbf{2 a}, \mathbf{b}$ and $\alpha-2 \mathbf{x}$ in good yields, and with no trace of $\beta$-DOBs regioisomers.

\section{Scheme 3. Plausible mechanism for the regioselective reduction of benzil 1a into DOB $\alpha$-2a using TMSCI/NaI combination in $\mathrm{CH}_{2} \mathrm{Cl}_{2}$}

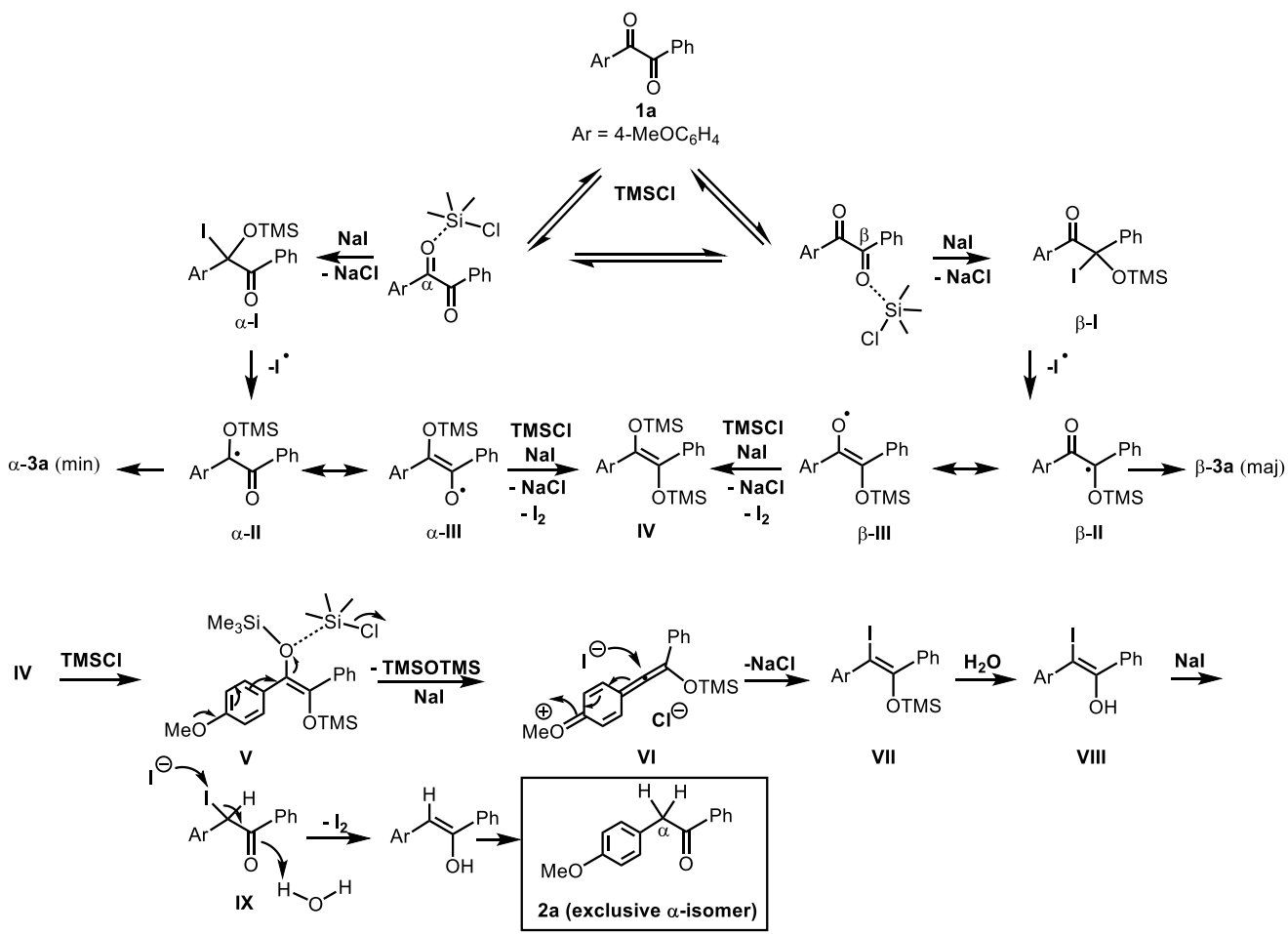

We next studied the spectroscopic data of the TMSCl/NaI combination by ${ }^{1} \mathrm{H}$ and ${ }^{13} \mathrm{C} \mathrm{NMR}$ in $\mathrm{CD}_{2} \mathrm{Cl}_{2}$. Under argon, a solution of equimolar proportions of $\mathrm{NaI}$ and $\mathrm{TMSCl}$ in $\mathrm{CD}_{2} \mathrm{Cl}_{2}$ furnished a colorless solution with absorptions at $\delta{ }^{1} \mathrm{H} 0.37 \mathrm{ppm}$ and $\delta{ }^{13} \mathrm{C} 1.34 \mathrm{ppm}$, rigorously identical with those of TMSCl alone (see ESI, Figure 1). On the contrary, TMSI in $\mathrm{CD}_{2} \mathrm{Cl}_{2}$ led to a pink-purple solution with different absorptions at 0.73 and $3.73 \mathrm{ppm}$. These observations strongly suggest that TMSI is not formed in methylene chloride by the combination of $\mathrm{TMSCl}$ with NaI, in agreement with Olah's report. ${ }^{1 b}$

To explain this unusual regioselective reduction of benzils 1 by the TMSCl/NaI combination in $\mathrm{CH}_{2} \mathrm{Cl}_{2}$, a plausible radical mechanism is proposed in Scheme 3. The reductive process begins probably by a coordination between oxygen atoms of 1a with TMSCl followed by a nucleophilic addition of $\mathrm{NaI}$ on the carbonyl functions giving intermediates $\alpha$-I and $\beta$-I. Then a radical fragmentation occurred on $\mathbf{I}$ to give siloxy ketone radical of type $\alpha$-II and $\beta$-II or their mesomeric forms $\alpha$-III and $\beta$ III. Further reaction of III in the presence of TMSCl and NaI would furnish the intermediate IV and $\mathrm{I}_{2}$, which coloured the methylene chloride solution in brown. ${ }^{16}$ Next, an additional equivalent of TMSCl would coordinate on the oxygen atom proximal to the electron-rich ring. The resulting intermediate $\mathbf{V}$ can lose TMSOTMS ${ }^{17}$ with the help of the $\mathrm{MeO}$ substituent to furnish species VI, thus explaining the regioselectivity of this reducing process. All our attempts to isolate TMSOTMS were unsuccessful however, when using $\mathrm{Ph}_{3} \mathrm{SiCl}$ instead of TMSCl,
$\mathrm{Ph}_{3} \mathrm{SiOSiPh}_{3}$ was successfully isolated after hydrolysis, reinforcing our ideas on this mechanism. A subsequent iodide atom addition on VI would give the alkenyl intermediate VII. After hydrolysis, the resulting enol VIII would react with NaI to provide $\alpha$-iodoketone IX which was reduced into $\mathbf{2 a}$ by an iodide. ${ }^{1 \mathrm{~b}, 18}$ To support this mechanistic hypothesis, incorporation of deuterium atoms into $\mathbf{2} \mathbf{a}$ in separate experiments was tried. No $\mathrm{D}_{2}$-labelled 2a was observed when using $\mathrm{CD}_{2} \mathrm{Cl}_{2}$ or $\mathrm{TMSCl}\left(\mathrm{D}_{9}\right)$. On the contrary, when $\mathbf{1 a}$ was reacted with TMSCl/NaI in $\mathrm{CH}_{2} \mathrm{Cl}_{2}$, hydrolysis with $\mathrm{D}_{2} \mathrm{O}$, allowed incorporation of two deuterium atoms on $\mathrm{C} \alpha$ of $\mathbf{2 a}$. To confirm that this selective transformation proceeded through a radical process rather than an anionic mechanism, ${ }^{19} \mathbf{1 z}$ having an o-allyl substituent, in which the terminal olefin would trap radical species, was reacted with $\mathrm{TMSCl} / \mathrm{NaI}$ in $\mathrm{CH}_{2} \mathrm{Cl}_{2}$ (Scheme 4). Accordingly, a cyclized product $\mathbf{4}$ was isolated after hydrolysis in $21 \%$ yield, together with unidentified by-products. This result strongly supports the radical mechanism ${ }^{20}$ depicted in Scheme 3.

Scheme 4. Reaction of $1 \mathrm{z}$ with $\mathrm{TMSCl} / \mathrm{NaI}$ combination in $\mathrm{CH}_{2} \mathrm{Cl}_{2}$ at $\mathrm{rt}$

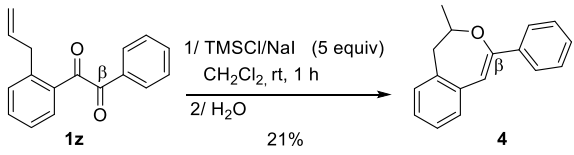

Because deoxybenzoins are important structural moieties that occur in bioactive molecules, ${ }^{21}$ it is now possible to have a rapid and efficient access to the corresponding $\alpha$ - and $\beta$-DOBs 2 starting from a single diarylalkyne using two different pathways 
(Scheme 5). As an illustration, diarylalkynes 5a-c were successfully transformed into $\beta-\mathbf{2 g}, \beta-2 n$ and $\beta-2 p$ after a regioselective metal-free hydration of the triple bond in the presence of a catalytic amount of PTSA in EtOH using a protocol developed in our group. ${ }^{22}$ On the other hand, diarylalkynes 5a-c were readily oxidized into their corresponding benzil derivatives $\mathbf{1 g}, \mathbf{1 n}$ and 1p using $\mathrm{DMSO}^{23}$ in the presence of catalytic amounts of $\mathrm{PdI}_{2}$. The reduction of these benzils by the TMSCl/NaI system, which was discussed above, led rapidly and efficiently to the corresponding DOBs $\alpha-2 \mathbf{g}, \alpha-2 \mathbf{n}$ and $\alpha-2 p$ with good yields and with a total $\alpha$-regioselectivity.

Scheme 5. Regioselective access to either $\alpha$-DOBs $2 \mathrm{~g}, 2 \mathrm{n}, 2 \mathrm{p}$ or $\beta$-DOBs 2g, 2n, 2p from diarylalkynes 5a-c

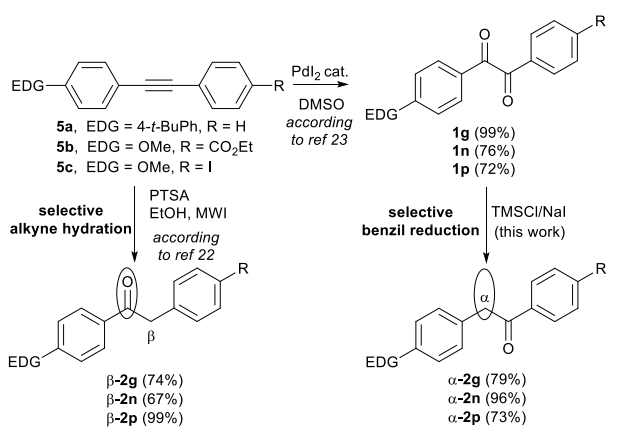

In summary, we have demonstrated for the first time that the TMSCl/NaI combination in $\mathrm{CH}_{2} \mathrm{Cl}_{2}$ is a highly efficient reducing system of unsymmetrical benzil derivatives. The easy to handle protocol developed herein provides a facile, rapid and regioselective access to DOBs $\alpha-2$ at rt with no need of any hazardous reagents or expensive metals. The reduction takes place invariably on the carbonyl proximal to the more electronrich aromatic ring. Since sustainable development is now a real challenge for the chemical industry, we think that this ecofriendly metal-free reduction is an important contribution in this field.

\section{ASSOCIATED CONTENT}

\section{Supporting Information}

Experimental procedure, NMR spectra, and analytical data for all new compounds (PDF)

\section{AUTHOR INFORMATION}

\section{Corresponding Author}

*olivier.provot@u-psud.fr and/or mouad.alami@u-psud.fr

\section{Author Contributions}

$\$$ These authors contributed equally.

\section{Notes}

The authors declare no competing financial interest.

\section{ACKNOWLEDGMENT}

Dr. Philippe Renaud, Univ. of Bern, is greatly thanked for helpful mechanism discussions. Authors gratefully acknowledge support of this project by CNRS, Univ. Paris-Sud, and by La Ligue Nationale Contre le Cancer through an Equipe Labellisée 2014 grant. LZY thanks the Chinese Scholarship Council for a Ph.D. funding.
Our laboratory is a member of the laboratory of excellence LERMIT supported by a grant from ANR (ANR-10-LABX-33).

\section{REFERENCES}

(1) (a) Olah, G.A.; Prakash, G.K.S.; Krishnamurti, R. In "Advances in Silicon Chemistry” G.L. Larson, Ed., JAI Press, London, 1991, pp.1. (b) Olah, G.A.; Narang, S.C. Tetrahedron 1982, 38, 2225 and refs therein.

(2) Hergott, H.H.; Simchen, G. Liebigs Ann. Chem. 1980, 1718.

(3) (a) Drabowicz, J.; Numata, T.; Oae, S. Org. Prep. Proced. Int. 1977, 9, 63. (b) Olah, G.A.; Gupta, B.G.B.; Narang, S.C. Synthesis 1977, 583.

(4) Magano, J.; Dunetz, J.R.; Org. Process Res. Dev. 2012, 16, 1156.

(5) Kuethe, J.T.; Childers, K.G.; Peng, Z.; Journet, M.; Humphrey, G.R.; Vickery, T.; Bachert, D.; Lam, T.T. Org. Process Res. Dev. 2009, 13, 576 .

(6) Flores-Gaspar, A.; Pinedo-Gonzalez, P.; Crestani, M.G.; Munoz-Hernandez, M.; Morales-Morales, D.; Warsop, B.A.; Jones, W.D.; Garcia, J.J. J. Mol. Catal. A-Chem. 2009, 309, 1.

(7) (a) Kamochi, Y.; Kudo, T.; Masuda, T.; Takadate, A. Chem. Pharm. Bull. 2005, 53, 1017. (b) Lin, W.; Hu, M.-H.; Feng, X.; Fu, L.; Cao, C.-P.; Huang, Z.-B.; Shi, D.-Q. Tetrahedron Lett. 2014, 55, 2238.

(8) (a) Japp, F.R.; Klingemann, F. J. Chem. Soc. Perkin Trans. 1893, 63, 770. (b) Fuson, R.C.; Hoch, P.E. J. Am. Chem. Soc. 1949, 71, 1585. (c) Fuson, R.C.; Armstrong, L.J.; Chadwick, D.H.; Kneisley, J.W.; Rowland, S.P.; Shenk, Jr, W.J.; Soper, Q.F. J. Am. Chem. Soc. 1945, 67, 386. (d) Ranganathan, T.; Cossette, P.; Emrick, T. J. Mater. Chem. 2010, 20, 3681. (e) Mayer, R.; Hiller, G; Nitzschke, M.; Jentzsch, J. Angew. Chem. Int. Ed. Engl. 1963, 2, 370.

(9) Identification of $\alpha-\mathbf{2 a}$ was determined by COSY, NOESY, HMBC and HSQC experiments.

(10) When 1a was treated by $\mathrm{HI}$ (wt 57\%) in refluxing $\mathrm{AcOH}$ according to Fuson's conditions (ref. 8b), $2 \mathbf{h}$ was obtained (80\%) indicating that a demethylation occured to provide a phenolic species.

(11) ${ }^{1} \mathrm{H}$ NMR results revealed that the crude consists of a mixture of $\alpha-2 a / \beta-3 a / \alpha-2 h$ in a $15 / 75 / 10$ ratio.

(12) Identification and ratio of regioisomers $\alpha-3 \mathbf{a} / \beta-3 \mathbf{a}$ was determined by COSY, NOESY, HMBC and HSQC experiments on the crude mixture.

(13) Zhang, W.; Shi, M. Chem. Commun. 2006, 1218.

(14) Mahmoodi, N.O.; Noori Navrood, M. Arkivoc 2007, 37.

(15) (a) Procuranti, B.; Connon, S.J. Chem. Commun. 2007, 1421. (b) Huang, Q.; Wu, J.-W.; Xu, H.-J. Tetrahedron Lett. 2013, 54, 3877.

(16) After hydrolysis, the brown $\mathrm{I}_{2}$-mixture was discolored by a saturated $\mathrm{Na}_{2} \mathrm{~S}_{2} \mathrm{O}_{3}$ solution.

(17) Ho, T.S. Synth. Commun. 1979, 9, 665.

(18) (a) Olah, G.A.; Arvanaghi, M.; Vankar, Y.D. J. Org. Chem. 1980, 45, 3531. (b) Peng, A-Y.; Ding, Y.-X. Org. Lett. 2004, 6, 1119.

(19) Addition in the media of TEMPO, a radical scavenger, completely inhibited the reducing process of the benzil derivative $\mathbf{1 a}$.

(20) Since a radical mechanism was supported by the transformation of $\mathbf{1 z}$ into 4 , we discarded the formation of intermediate IV through a reaction of $\alpha$-I or $\beta$-I with an iodine atom.

(21) (a) Hu, Q.-F.; Zhou, B.; Ye, Y.-Q.; Jiang, Z.-Y.; Huang, X.-Z.; Li, Y.-K.; Du, G.; Yang, G.-Y.; Gao, X.-M. J. Nat. Prod. 2013, 76, 1854. (b) Li, H.-Q.; Xue, J.-Y.; Shi, L.; Gui, S.-Y.; Zhu, H.-L. Eur. J. Med. Chem. 2008, 43, 662. (c) Lu, T.-M.; Kuo, D.-H.; Ko, H.-H.; Ng, L.-T. J. Agric. Food. Chem. 2010, 58, 10027.

(22) G. Le Bras, O. Provot, J.-F. Peyrat, M. Alami, J.-D. Brion, Tetrahedron Lett., 2006, 47, 5497.

(23) C. Mousset, O. Provot, A. Hamze, J. Bignon, J.-D.; Brion, M. Alami, Tetrahedron, 2008, 64, 4287. 